\title{
Rifaximin for the Management of Colonic Diverticular Disease: far Beyond a Simple Antibiotic
}

\author{
Antonio Tursi ${ }^{1}$, Carmelo Scarpignato ${ }^{2}$, Giovanni Brandimarte ${ }^{3}$, Francesco Di Mario ${ }^{4}$, Angel Lanas ${ }^{5}$
}

1) Gastroenterology Service,

ASL BAT, Andria, Italy

2) Clinical Pharmacology \&

Digestive Pathophysiology

Unit, Department of Medicine

\& Surgery, University of

Parma, Italy

3) Division of

Internal Medicine and

Gastroenterology, „Cristo Re”

Hospital, Rome, Italy

4) Gastroenterology Unit,

Department of Medicine \&

Surgery, University of Parma,

Parma, Italy

5) Service of Digestive

Diseases, University Clinic

Hospital Lozano Blesa

University of

Zaragoza, IIS Aragón,

Zaragoza, Spain

Address for correspondence:

Antonio Tursi, MD

Via Torino 49,

76123 Andria (BT), Italy

antotursi@tiscali.it
Received: 30.10 .2018

Accepted: 26.11.2018
Diverticulosis of the colon is one of the most common anatomic alterations occurring in the human colon, characterized by the presence of sac-like protrusions called "diverticula" [1].

Diverticulosis in the western world is generally left-sided, with herniation involving only mucosa and submucosa, largely age-dependent and increasing up to $65 \%$ in those aged 65 years or more [2]. Only about $20 \%$ of those patients will develop symptoms, the so-called "Diverticular Disease"(DD) [1], of whom $15 \%$ will ultimately develop diverticulitis [3], with or without complications.

\section{Clinical pictures of diverticular disease}

From a clinical standpoint, DD is currently subdivided as Symptomatic Uncomplicated Diverticular Disease (SUDD), recurrent symptomatic disease and complicated disease [4] SUDD is characterized by leftlower quadrant pain lasting $>24 \mathrm{~h}$, not fulfilling the Rome criteria for IBS diagnosis and sometimes associated with raised levels of fecal calprotectin (FC) $[5,6]$. These features are therefore useful to allow a correct differential diagnosis between IBS and SUDD in patients harboring colonic diverticula [7].

The development of inflammation in diverticula results in acute diverticulitis. It has been suggested that fecal matter becomes trapped in the diverticula and as a result, low-grade inflammation develops due to abrasion of the mucosa, allowing access of the fecal microbiota to the lamina propria, leading to acute inflammation of the mucosa, which usually begins at the apex of the sac [8]. This can be associated with acute inflammation of the mesenteric and pericolic fat with the formation of a diverticular abscess. Another postulated mechanism for the development of acute diverticulitis is a microperforation at the fundus of the diverticulum leading to inflammation [8]. Very recently, an ischemic pathogenesis has been hypothesized [9]. The trigger of the ischemic process may be a long-lasting (or recurrent) compression of vasa recta in the "neck" of the diverticulum due to a prolonged and marked contractile activity of the colon. Indeed, the tiny "neck" of the diverticulum passes through the circular muscle of the bowel wall and could be abnormally compressed, leading to mucosal ischemia at the apex of the sac or even microperforation. The "traumatic" and "ischemic" mechanisms of acute diverticulitis may not be mutually exclusive since they may act in different patients (old versus young) or in different types (large versus diminutive) diverticula [9].

\section{Diverticular disease: which treatment?}

According to the statements of the 2nd International Congress on Diverticular Disease [10], several pharmacological approaches targeting enteric bacteria (with poorly absorbed antibiotics, such as rifaximin, or probiotics) and/or intestinal inflammation (with 5-ASA derivatives or rifaximin) have shown to be capable of controlling symptoms and also preventing complications in patients with SUDD. No treatment is advised in patients with asymptomatic diverticulosis, but a high fiber diet is suggested to prevent the development of further diverticula.

The rationale for the use of poorly absorbed antibiotics relies on the presence of small intestinal bacterial overgrowth (SIBO, the most widely characterized form of dysbiosis) in patients with DD and its role in symptom generation [11]. Bacteria-induced immune activation will drive low-grade mucosal inflammation, which sensitizes both intrinsic primary efferent and extrinsic primary afferent neurons generating neural and smooth muscle dysfunction. These disturbances will lead to symptom development and/or persistence [11].

In this issue, Experts from the Romanian Society of Gastroenterology provide updated guidelines for the 
management of patients with DD [12]. Like many other guidelines [13-19], the Romanian Position Paper recommends in patients with SUDD the use of cyclic rifaximin to reduce the severity and frequency of abdominal symptoms.

Rifaximin: peculiar features of a gastro-intestinal (GI)targeted, poorly absorbed antibiotic

Rifaximin is a non-aminoglycoside, semisynthetic, non-systemic antibiotic derived from rifamycin SV. It was originally licensed in Italy as polymorphic form a in 1985 for the treatment of several GI diseases, and was later approved in 33 countries under different trade names [20].

Rifaximin inhibits bacterial protein synthesis by binding to the $\beta$-subunit of bacterial DNA-dependent RNA polymerase. This activity leads to the suppression of RNA chain initiation during RNA synthesis [21]. In vitro and in vivo, it shows a strong activity against Gram-positive and Gram-negative bacteria, both aerobic and anaerobic [20, 21]. In addition, thanks to a very low systemic absorption [22], its safety profile is excellent, since adverse events were observed in less than $2 \%$ of patients $[23,24]$. Most of the side effects were GI (such as nausea, vomiting, meteorism, abdominal pain), while skin rash was rare [24]. Rifaximin was originally approved for traveler's diarrhea and overt hepatic encephalopathy. However, since this antibiotic is very effective in eradicating SIBO [25], which mirrors the underlying dysbiosis of many organic and functional GI disorders [26], its use has been extended to irritable bowel syndrome (IBS), inflammatory bowel disease (IBD) as well as DD. The microbiota-modulating effects (often referred to as eubiotic effects) and the intrinsic anti-inflammatory activity likely explain the observed clinical efficacy of this antibiotic in all the above clinical conditions, where bacteria and inflammation exert a pathogenic role [11].

\section{Antibiotic activity}

In vitro rifaximin exerts antimicrobial activity against Gram-positive and Gram-negative bacteria, both aerobic and anaerobic. The minimal inhibitory concentration for $90 \%$ of test strains $\left(\mathrm{MIC}_{90}\right)$ of Escherichia coli and other coliforms, including most non-strict anaerobic bacterial enteropathogens, ranges from 16 to $32 \mu \mathrm{g} / \mathrm{ml}$ [27]. Moreover, the drug can reduce the virulence of bacterial enteropathogens, as enterotoxigenic Escherichia coli (ETEC) and enteroaggregative Escherichia coli (EAEC) and Shigella [28, 29].

Rifaximin is usually given cyclically (7-10 days every month). The rational for this kind of administration relies on several reasons:

- SIBO is a chronic, relapsing condition and the antimicrobial effects are short-lasting. Indeed, after a 5-day treatment of healthy volunteers with this poorly absorbable antibiotic at a dose of $800 \mathrm{mg} /$ day, the decrease of bacterial counts is limited to the first two weeks after treatment, after which it gradually recovers $[24,30]$. Along the same lines, after rifaximin (given at $1800 \mathrm{mg} /$ day in 3 treatment periods of 10 days each followed by 25 -day washouts) therapy in patients with ulcerative colitis intestinal bacterial load returned to initial values after each washout period [31].

- Cyclic rifaximin treatment permits the prevention of colonization with resistant bacterial strains, which can develop after rifaximin therapy (albeit the frequency is very low), but which can disappear rapidly after the cessation of the drug treatment [30].

- The washout rifaximin period will allow probiotics (preferably a Lactobacilli-Bifidobacteria mixture) filling the open ecologic niche, thereby improving the intestinal microecology towards a healthy microbiota [11].

\section{Anti-inflammatory activity}

Rifaximin exerts a significant anti-inflammatory effect, reducing gut inflammation and reestablishing an effective epithelial mucosal barrier. These anti-inflammatory properties have been demonstrated for the first time in a study in which epithelial cells, exposed to sub-therapeutic concentrations of rifaximin, showed a reduction in basal levels of inflammatory cytokines [31]. Rifaximin may also exert beneficial effects in specific GI disorders through gut-specific activation of the pregnane $\mathrm{X}$ receptor (PXR) [32, 33], a nuclear receptor and transcription factor [34]. Indeed, in vitro and in vivo studies in epithelial cell lines demonstrated that rifaximin also displayed a potent PXR-dependent anti-inflammatory activity [35]. Alteration of levels of PXR are associated with activity in IBD and may explain the beneficial effect of rifaximin in this clinical setting [36, 37].

Rifaximin can modulate the bacterial adhesion to the intestinal mucosa and, consequently, the release of inflammatory cytokines and the expression of nuclear factor$\kappa \mathrm{B}(\mathrm{NF}-\kappa \mathrm{B})$. As a member of the rifamycin class, it belongs to the ansamycins family, which has well-established antiinflammatory and immunosuppressive properties [37]. In this connection, rifaximin was found capable of reducing bacterial-related activation and modulating the adhesion molecules expression patterns, associated with T-cell homing to the intestinal mucosa [38].

A recent experimental study [39] confirmed the antiinflammatory activity of rifaximin in a model of diclofenacinduced intestinal inflammation, where the drug decreased the myeloperoxidase (MPO) and cytokine tissue concentrations as well as the fecal excretion of calprotectin. In-depth investigations also showed that - in a human monocyte cell line (THP-1), a well-established model to study monocyte/ macrophage functions - this antibiotic is able to modulate the activity of the inflammasome NLRP3 (nucleotide-binding oligomerization domain leucine rich repeat and pyrin domaincontaining protein 3 ) by preventing the caspase-1-dependent activation and release of interleukin-1 $\beta$ (IL-1 $\beta$ ) [39]. This original observation provides compelling evidence for an intrinsic anti-inflammatory activity of rifaximin (Fig. 1).

\section{Eubiotic effects}

One of the most intriguing activities of rifaximin is represented by its effect on gut microbiota composition. Early data showed that the overall gut microbiota composition is not affected by rifaximin treatment [40-42]. Subsequent studies indicated that rifaximin treatment promotes the growth of beneficial bacteria, such as Bifidobacteria and Lactobacilli. Recent observations support the hypothesis that rifaximin exerts a beneficial modulation of colonic microflora, namely an "eubiotic" effect [43]. Ponziani et al. [44] treated 20 patients with different GI and liver diseases with a 10-day course of rifaximin $1,200 \mathrm{mg} /$ day. A culture-independent metagenomic analysis was carried out on fecal samples at the beginning of 


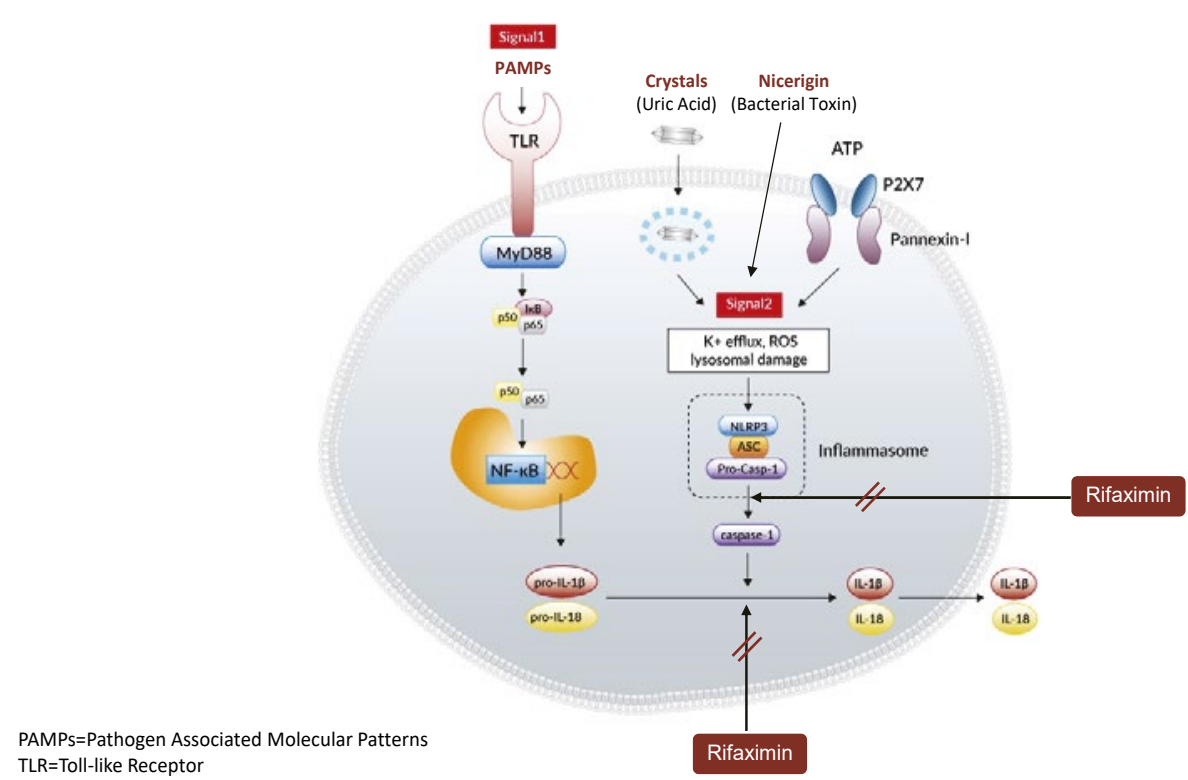

Fig. 1. Anti-inflammatory activity of rifaximin: Modulation of the inflammasome functions and signaling pathways. In THP-1 cells, primed with LPS, the antibiotic prevents nigericin-induced activation and release of IL-1b by reducing the conversion of pro-caspase-1 into caspase-1 [39].

the study, at the end of treatment and 30 days thereafter. A significant decrease in Roseburia, Haemophilus, Veillonella and Streptococcus abundance was observed after 10 days of rifaximin administration as well as a reduction in Roseburia during the follow-up period, while a significant increase in Lactobacilli abundance after drug treatment was evident, an effect persisting after stopping the treatment [44].

This significant effect on Lactobacilli could have important clinical consequences. Indeed, this species has been reported to down-regulate pro-inflammatory cytokines and TNF production, to inhibit translocation of pathogenic bacteria and to restore impaired intestinal permeability [45-49].

\section{Conclusions}

The rationale underlying rifaximin use in DD relies on its "pleiotropic" pharmacologic activities, rendering it a unique GI-targeted antibiotic. Its eubiotic effects, coupled with its antiinflammatory activity, make it particularly suitable for a condition such as DD, where dysbiosis and mucosal inflammation are common and represent important pathogenic mechanisms for the development of both symptoms and complications. Its safety (due to the negligible systemic absorption) and the minimal, if any, risk of development of bacterial resistance (even after prolonged administration) are additional benefits.

The efficacy of long-term cyclic administration of rifaximin in SUDD has been evaluated in several long-term (12-24 months) trials [50], and its use in preventing recurrence of acute diverticulitis appears promising [51]. Specifically designed dose-ranging studies, as well as clinical trials assessing treatments of different duration are now mandatory to select the best regimen (dose and duration) for patients with DD.

Conflicts of interest: C.S. is member of the Advisory Board and the Speakers' Bureau of Alfasigma. A.L. is member of the Speakers' Bureau of AlfaSigma. The other authors declare that they have no conflicts of interest concerning this article.
Authors' contributions: A.T.: conception and design of the editorial, acquisition of data, analysis and interpretation of data. A.T., G.B., A.L., F.M., C.S.: drafting the manuscript and revising it critically for important intellectual content. A.T., G.B, A.L., F.M., C.S.: final approval of the version to be submitted.

\section{REFERENCES}

1. Tursi A, Papagrigoriadis S. Review article: the current and evolving treatment of colonic diverticular disease. Aliment Pharmacol Ther 2009;30:532-546. doi:10.1111/j.1365-2036.2009.04072.x

2. Tursi A. Diverticulosis today: unfashionable and still underresearched. Therap Adv Gastroenterol 2016;9:213-228. doi:10.1177/1756283X15621228

3. Elisei W, Tursi A. The Pathophysiology of Colonic Diverticulosis: Inflammation versus Constipation? Inflamm Intest Dis 2018 Jun 28. doi: $10.1159 / 000489173$

4. Kohler L, Sauerland S, Neugebauer E, et al. Diagnosis and treatment of diverticular disease: result of a consensus development conference. The Scientific Committee of the European Association for Endoscopic Surgery. Surg Endosc 1999;13:430-436. doi:10.1007/s004649901007

5. Tursi A, Elisei W, Picchio M, Giorgetti GM, Brandimarte G. Moderate to severe and prolonged left lower-abdominal pain is the best symptom characterizing symptomatic uncomplicated diverticular disease of the colon: a comparison with fecal calprotectin in clinical setting. J Clin Gastroenterol 2015;49:218-221. doi:10.1097/MCG.0000000000000094

6. Cuomo R, Barbara G, Andreozzi P, et al. Symptom patterns can distinguish diverticular disease from irritable bowel syndrome. Eur J Clin Invest 2013;43:1147-1155. doi:10.1111/eci.12152

7. Tursi A. Diverticular disease of the colon and irritable bowel syndrome it is time to differentiate. Am J Gastroenterol 2015;110:774-775. doi:10.1038/ajg.2015.78

8. Tursi A, Papa A, Danese S. Review article: the pathophysiology and medical management of diverticulosis and diverticular disease of the colon. Aliment Pharmacol Ther 2015;42:664-684. doi:10.1111/apt.13322 
9. Zullo A. Medical hypothesis: speculating on the pathogenesis of acute diverticulitis. Ann Gastroenterol 2018;31:747-749. doi:10.20524/ aog.2018.0315

10. Tursi A, Picchio M, Elisei W, Di Mario F, Scarpignato C, Brandimarte G. Management of Patients With Diverticulosis and Diverticular Disease: Consensus Statements From the 2nd International Symposium on Diverticular Disease. J Clin Gastroenterol 2016;50 (Suppl 1):S101-S107. doi:10.1097/MCG.0000000000000654

11. Scarpignato C, Barbara G, Lanas A, Strate LL. Management of colonic diverticular disease in the third millennium: Highlights from a symposium held during the United European Gastroenterology Week 2017. Therap Adv Gastroenterol 2018;11:1756284818771305. doi: $10.1177 / 1756284818771305$

12. Trifan A, Gheorghe C, Sabo CM, et al. Diagnosis and Treatment of Colonic Diverticular Disease: Position Paper of the Romanian Society of Gastroenterology and Hepatology. J Gastrointestin Liver Dis 2018; 27: 449-457. doi: 10.15403/jgld.2014.1121.274.rom

13. Charua-Guindic L, Mazza-Olmos D, Orduna-Tellez D, et al Gastroenterology. Diagnosis and treatment guidelines of diverticular disease of the colon. Treatment. Rev Gastroenterol Mex 2008;73:261 264.

14. Andersen JC, Bundgaard L, Elbrond H, et al. Danish national guidelines for treatment of diverticular disease. Dan Med J 2012;59:C4453.

15. Kruis W, Germer CT, Leifeld L, et al. Diverticular disease: guidelines of the german society for gastroenterology, digestive and metabolic diseases and the german society for general and visceral surgery. Digestion 2014;90:190-207. doi:10.1159/000367625

16. Cuomo R, Barbara G, Pace F, et al. Italian consensus conference for colonic diverticulosis and diverticular disease. United European Gastroenterol J 2014; 2: 413-442. doi:10.1177/2050640614547068

17. Vennix S, Morton DG, Hahnloser D, et al. Systematic review of evidence and consensus on diverticulitis: an analysis of national and international guidelines. Colorectal Dis 2014;16:866-878. doi:10.1111/codi.12659

18. Pietrzak A, Bartnik W, Szczepkowski M, et al. Polish interdisciplinary consensus on diagnostics and treatment of colonic diverticulosis (2015) Pol Przegl Chir 2015;87:203-220. doi:10.1515/pjs-2015-0045

19. Binda GA, Cuomo R, Laghi A, et al. Practice parameters for the treatment of colonic diverticular disease: Italian Society of Colon and Rectal Surgery (SICCR) guidelines. Tech Coloproctol 2015;19:615-626. doi:10.1007/s10151-015-1370-X

21. Scarpignato C, Pelosini I. Rifaximin, a poorly absorbed antibiotic: pharmacology and clinical potential. Chemotherapy 2005;51(Suppl 1):36-66. doi:10.1159/000081990

22. Scarpignato C, Pelosini I. Experimental and clinical pharmacology of rifaximin, a gastrointestinal selective antibiotic. Digestion 2006;73(Suppl 1):13-27. doi:10.1159/000089776

23. Blandizzi C, Viscomi GC, Marzo A, Scarpignato C. Is generic rifaximin still a poorly absorbed antibiotic? A comparison of branded and generic formulations in healthy volunteers. Pharmacol Res 2014;85:39-44. doi:10.1016/j.phrs.2014.05.001

24. Shah E, Kim S, Chong K, Lembo A, Pimentel M.. Evaluation of harm in the pharmacotherapy of irritable bowel syndrome. Am J Med 2012;125:381-393. doi:10.1016/j.amjmed.2011.08.026

25. Gillis JC, Brogden RN. Rifaximin. A review of its antibacterial activity, pharmacokinetic properties and therapeutic potential in conditions mediated by gastrointestinal bacteria. Drugs 1995;49:467-484. doi:10.2165/00003495-199549030-00009

26. Gatta L, Scarpignato C. Systematic review with meta-analysis: rifaximin is effective and safe for the treatment of small intestine bacterial overgrowth. Aliment Pharmacol Ther 2017;45:604-616. doi:10.1111/ apt. 13928

27. Scarpignato C, Gatta L. Commentary: Towards an effective and safe treatment of small intestine bacterial overgrowth. Aliment Pharmacol Ther 2013;38:1409-1410. doi:10.1111/apt.12531

28. Gomi H, Jiang ZD, Adachi JA, et al. In vitro antimicrobial susceptibility testing of bacterial enteropathogens causing traveler's diarrhea in four geographic regions. Antimicrob Agents Chemother 2001;45:212-216. doi:10.1128/AAC.45.1.212-216.2001

29. Jiang ZD, Ke S, DuPont HL. Rifaximin-induced alteration of virulence of diarrhoea-producing Escherichia coli and Shigella sonnei. Intl J Antimicrob Agents 2010;35:278-281. doi:10.1016/j. ijantimicag.2009.11.013

30. Brown EL, Xue Q, Jiang ZD, Xu Y, Dupont HL. Pretreatment of epithelial cells with rifaximin alters bacterial attachment and internalization profiles. Antimicrob Agents Chemother 2010;54:388-396. doi:10.1128/ AAC.00691-09

31. De Leo C, Eftimiadi C, Schito GC. Rapid disappearance from the intestinal tract of bacteria resistant to Rifaximin. Drugs Exp Clin Res 1986;12:979-981.

32. Brigidi P, Swennen E, Rizzello F, Bozzolasco M, Matteuzzi D. Effects of rifaximin administration on the intestinal microbiota in patients with ulcerative colitis. J Chemother 2002;14: 290-295. doi:10.1179/ joc.2002.14.3.290

33. Ma X, Shah YM, Guo GL, et al. Rifaximin is a gut-specific human pregnane X receptor activator. J Pharmacol Exp Ther 2007;322:391-398.

34. Esposito G, Nobile N, Gigli S, et al. Rifaximin improves Clostridium difficile toxin A-induced toxicity in Caco- 2 cells by the PXR-dependent TLR4/MyD88/NF-kB pathway. Front Pharmacol 2016;9:120. doi:10.3389/fphar.2016.00120

35. Cheng J, Shah YM, Gonzalez FJ. Pregnane X receptor as a target for treatment of inflammatory bowel disorders. Trends Pharmacol Sci 2012;33:323-330. doi:10.1016/j.tips.2012.03.003

36. Dring MM, Goulding CA, Trimble VI, et al. The pregnane X receptor locus is associated with susceptibility to inflammatory bowel disease. Gastroenterology 2006;130:341-348. doi:10.1053/j. gastro.2005.12.008

37. Terc J, Hansen A, Alston L, Hirota SA. Pregnane X receptor agonists enhance intesti-nal epithelial wound healing and repair of the intestinal barrier followingthe induction of experimental colitis. Eur J Pharm Sci 2014;55:12-19. doi:10.1016/j.ejps.2014.01.007

38. Labro MT. Anti-inflammatory activity of ansamycins. Exp Rev Ant Infect Ther 2005;3:91-103. doi:10.1586/14787210.3.1.91

39. Cianci R, Frosali S, Pagliari D, et al. Uncomplicated diverticular disease: innate and adaptive immunity in human gut mucosa before and after rifaximin. J Immunol Res 2014;2014:696812. doi:10.1155/2014/696812

40. Colucci R, Pellegrini C, Fornai M, et al. Pathophysiology of NSAIDassociated intestinal lesions in the rat: luminal bacteria and mucosal inflammation as targets for prevention. Front Pharmacol 2018;9:1340.

41. Kim MS, Morales W, Hani AA, et al. The effect of rifaximin on gut flora and Staphylococcus resistance. Dig Dis Sci 2013;58:1676-1682. doi:10.1007/s10620-013-2675-0

42. Maccaferri S, Vitali B, Klinder A, et al. Rifaximin modulates the colonic microbiota of patients with Crohn's disease: an in vitro approach using a continuous culture colonic model system. J Antimicrob Chemother 2010;65:2556-2565. doi:10.1093/jac/dkq345

43. Bajaj JS, Heuman DM, Sanyal AJ, et al. Modulation of the metabiome by rifaximin in patients with cirrhosis and minimal hepatic encephalopathy PLoS One 2013;8:e60042. doi:10.1371/journal.pone.0060042 
44. Ponziani FR, Zocco MA, D’Aversa F, Pompili M, Gasbarrini A. Eubiotic properties of rifaximin: Disruption of the traditional concepts in gut microbiota modulation. World J Gastroenterol 2017;23:4491-4499. doi:10.3748/wjg.v23.i25.4491

45. Ponziani FR, Scaldaferri F, Petito V et al.The role of antibiotics in gut microbiota modulation: the eubiotic effects of rifaximin. Dig Dis 2016; 34: 269-278.

46. Borruel N, Carol M, Casellas F, et al. Increased mucosal tumour necrosis factor alpha production in Crohn's disease can be downregulated ex vivo by probiotic bacteria. Gut 2002;51: 659-664. doi:10.1136/gut.51.5.659

47. Thomas CM, Hong T, van Pijkeren JP, et al. Histamine derived from probiotic Lactobacillus reuteri suppresses TNF via modulation of PKA and ERK signaling. PLoS One 2012;7:e31951. doi:10.1371/journal. pone.0031951

48. Llopis M, Antolin M, Carol M, et al. Lactobacillus caseidownregulates commensals' inflammatory signals in Crohn's disease mucosa. Inflamm Bowel Dis 2009;15:275-283. doi:10.1002/ibd.20736
49. Ait-Belgnaoui A, Bradesi S, Fioramonti J, Theodorou V, Bueno L. Acute stress-induced hypersensitivity to colonic distension depends upon increase in paracellular permeability: role of myosin light chain kinase. Pain 2005;113:141-147. doi:10.1016/j.pain.2004.10.002

50. Zareie M, Johnson-Henry K, Jury J, et al. Probiotics prevent bacterial translocation and improve intestinal barrier function in rats following chronic psychological stress. Gut 2006;55:1553-1560. doi:10.1136/ gut.2005.080739

51. Bianchi M, Festa V, Moretti A, et al. Meta-analysis: long-term therapy with rifaximin in the management of uncomplicated diverticular disease. Aliment Pharmacol Ther 2011;33:902-910. doi:10.1111/j.13652036.2011.04606.x

52. Lanas A, Ponce J, Bignamini A, Mearin F. One year intermittent rifaximin plus fibre supplementation vs. fibre supplementation alone to prevent diverticulitis recurrence: a proof-of-concept study. Dig Liver Dis 2013;45:104-109. doi:10.1016/j.dld.2012.09.006 